\title{
Radiative colliding winds models: the stagnation point singularity
}

\author{
Svetozar A. Zhekov \\ JILA, University of Colorado, Boulder, CO 80309-0440, USA
}

\author{
A.V. Myasnikov and N.A. Belov \\ Institute for Problems in Mechanics, Russian Academy of Sciences \\ 101 Vernadskii Ave., Moscow 117526, Russia
}

\begin{abstract}
The singularity at the stagnation point in steady-state colliding winds has a big influence on the structure of the radiative interaction region. None of the existing numerical models treats properly this mathematical problem. As a result, all the available models cannot be used for deriving the stellar winds parameters by making a comparison with the observed X-ray properties.
\end{abstract}

\section{Discussion}

The singularity problem at the stagnation point of radiative steady-state colliding winds was first addressed by Myasnikov \& Zhekov (1993). For a corresponding detailed study we refer to Myasnikov et al. (1998). It was shown that all the available numerical models are not suitable for studying the stability properties of these flows. On the other hand, we would like to emphasize here another important corollary from this study. Namely, that the X-ray properties of the interaction region are very dependent on how the singularity was eliminated. For example, the theoretical X-ray luminosity of V444 Cyg can differ by one order of magnitude. But, this number should not be considered as typical since this difference can be any. This is why it is straightforward to conclude that: The available numerical models for the radiative steady-state colliding winds cannot be used for deriving the stellar winds parameters by confronting the model predictions with observations. All the parameters derived until now by using this technique have practically no value.

Acknowledgments. SAZh acknowledges the financial support by the NSF grant AST-9528256. AVM and NAB thank the Russian Foundation of Basic Research for the financial support under Grant No. 96-01-00738.

\section{References}

Myasnikov, A.V., Zhekov, S.A. 1993, MNRAS 260, 221

Myasnikov, A.V., Zhekov, S.A. Belov, N.A. 1998, MNRAS 298, 1021 